\title{
Optimization of Machining Parameters for AISI 316L And 317L Austenitic Stainless Steels using Eco-Cut Wire-EDM Technique
}

\author{
M.V.N. Srujan Manohar, Y. Seetha Rama Rao, Ch. Sree Ram
}

\begin{abstract}
Austentic stainless steel is one of the most suitable engineering material based on their superior resistance to corrosion and compatibility at high temperatures and high vacuum. However, the machinability of austenitic stainless steel is not very promising owing to lower thermal conductivity, higher degree of ductility and work hardenability. For meeting these challenges, unconventional machining procedures were evolved and can make any impenetrable design/profile on any work substance by acceptable controlling of various machining procedures. The main importance of this paper is to show the impact of machining parameters on Eco-cut Wire Electric Discharge Machining (WEDM) for disparate austenitic stainless steels (AISI 316L \& 317L). Initially both the metals are machined on WEDM. Machining parameters like pulse on time $\left(P_{\text {on }}\right)$, pulse off time $\left(P_{\text {off }}\right)$, voltage $(V)$ and wire tension(WT) are observed for both $316 \mathrm{~L}$ and $317 \mathrm{~L}$ stainless steel materials. A Box-Behnken Design (BBD) of response surface methodology (RSM) has been used for experimental work. The reaction of procedure is estimated by ANOVA analysis and response optimizer is used for optimum level checking. A series of trial runs were carried out on both the machined specimens for identifying better material removal rate(MRR), cutting speed(CS) and surface roughness $(\mathrm{Ra})$.
\end{abstract}

Keywords - Cutting Speed(CS), Material Removal Rate(MRR), Surface Roughness $(R a)$, pulse on time $\left(P_{o n}\right)$, pulse off time $\left(P_{\text {off }}\right)$, voltage $(V)$, wire tension(WT), Response Surface Methodology(RSM) and ANOVA.

\section{INTRODUCTION}

Modern advances in aerospace and nuclear engineering industries is partly reflects to the use of impenetrable-tomachine materials like alloys, nimonics, carbides, stainless steel etc. Many of these substances found various applications in the

industrial fields by exploiting their elevated strength-toweight relationship, hardness and heat-

resisting qualities. Conventional machining processes instead of present technological improvement it is impenetrable to machine these substances from the perspective of low-cost production. Un-conventional machining procedures are necessary to control these hard substances. Electrical discharge machining(EDM) is the most favored non-traditional material removal procedures and enhances basic principles of machining in manufacturing industries like aerospace, automotive, nuclear, medical and die-mould production.

Revised Manuscript Received on December 15, 2019.

M.V.N. Srujan Manohar, Research Scholar, Department of Mechanical Engineering, Pondicherry Engineering College, Puducherry, India. Email: srujansujith8990@gmail.com

Dr. Y. Seetha Rama Rao, Associate Professor, Department of Mechanical Engineering, GVPCOE (A), Visakhapatnam, India.

Email: yseetharamarao24@gvpce.ac.in

Ch. Sreeram, PG student, Department of Mechanical Engineering, GVPCOE (A), Visakhapatnam, India.

Email: powermanu555@gmail.com
Crucial advances of EDM was noticed when computer numerical influence apparatus were tried for machine tool production. Thus, EDM procedure became automatic and unattended machining. Klocke et al [1] explained about the structure and composition of the white layer In the WireEDM process. In EDM procedure thermal (heat) energy is pre-owned to fabricate heat that liquefies and vaporizes work piece by ionization within dielectric medium. The electrical discharges fabricate impulsive pressure by dielectric explosion to detach liquefied material.

Thus, all detached material can be effectively made to fabricate impenetrable \& precisely machine components. Liquefied material is not completely flushed away but remaining material resolidifies to form discharge craters. EDM processes are categorized into die-sinking EDM \& Wire- EDM. Electrode of die-sinking EDM has reversed design/profile of part to be machined, while Wire-EDM utilizes thin wire, varying from $0.01-0.36 \mathrm{~mm}$ in diameter, as electrode. WEDM was first applied in manufacturing production in the late 1960s. The advancement of the procedure was the denouement of seeking an approach to restore the machined electrode pre-owned in EDM. In 1974, D.H. Dulebohn claimed the optical-line follower apparatus to automatically impact the design/profile of the element/component to be machined by the WEDM procedure. As an outcome, the broad facility of WEDM procedure was extensively exploited for any through-hole machining payable to the wire, which has to pass between the parts to be machined. Several mathematical models are incorporated by researchers to optimize the machine parameters of WEDM [2],[3].

\section{FUNDAMENTAL WORKING OF WEDM PROCEDURE}

WEDM is a procedure of material removal of electrical conductive materials by thermo-electric source of energy. The material removal is by commanded erosion between a series/run of repetitive flash/twinkles in between electrodes, i.e. work piece and tool. In WEDM, the erosion apparatus has been explained as liquefying and/or evaporation of surface material by heat fabricated in the plasma channel. A flash is fabricated in between wire electrode and work piece through deionized water and erodes work piece to fabricate complex 2D and 3D object profiles.

\section{MATERIAL AND EXPERIMENTAL WORK}

Austenitic stainless steels (AISI 316L \& 317L) are preowned in this work [4],[5] and the specifications of Wire EDM Machine is shown in Table I and levels of input limitations is shown in Table II. 


\section{Optimization of Machining Parameters for AISI 316L And 317L Austenitic Stainless Steels using Eco-Cut Wire-EDM Technique}

The dimensions of the work piece for the work is taken as, Plates of $100 \times 150 \times 5 \mathrm{~mm}$ thick respectively, and these plates were machined by Wire EDM shown in Fig. 1.

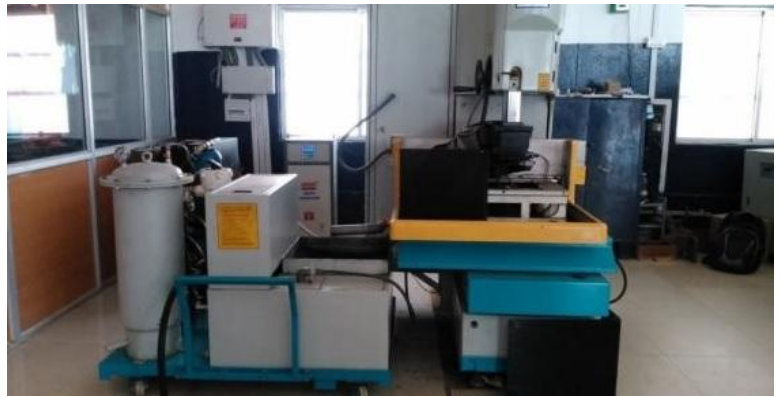

Fig. 1. Eco-cut Wire EDM Machine

Table-I: Specifications of Wire EDM machine

\begin{tabular}{|c|c|}
\hline Traverse & $250 \times 350 \times 200 \mathrm{~mm}$ \\
\hline Taper & $+/-5^{0}$ on $100 \mathrm{~mm}$ job height \\
\hline Max Cutting Speed & $\begin{array}{c}702 \mathrm{~mm} / \mathrm{min} \text { with } ø 0.25 \text { special } \\
\text { brass wire on } 50 \mathrm{~mm} \text { thick } \\
\text { HCHCR }\end{array}$ \\
\hline Surface Finish & $1.2 \mu \mathrm{Ra}$ \\
\hline
\end{tabular}

Table-II: Levels of input limitations

\begin{tabular}{|c|c|c|c|c|}
\hline Limitations & $\begin{array}{c}\text { Nota- } \\
\text { tion }\end{array}$ & $\begin{array}{c}\text { Level } \\
\mathbf{1}\end{array}$ & $\begin{array}{c}\text { Level } \\
\mathbf{2}\end{array}$ & $\begin{array}{c}\text { Level } \\
\mathbf{3}\end{array}$ \\
\hline Pulse on Time $\mathrm{P}_{\text {on }}(\mu \mathrm{s})$ & $\mathrm{A}$ & 112 & 113 & 114 \\
\hline Pulse off Time $\mathrm{P}_{\text {off }}(\mu \mathrm{s})$ & $\mathrm{B}$ & 44 & 45 & 46 \\
\hline Voltage V $(\mathrm{V})$ & $\mathrm{C}$ & 20 & 22 & 24 \\
\hline Wire TensionWT $(\mathrm{m} / \mathrm{min})$ & $\mathrm{D}$ & 9 & 10 & 11 \\
\hline
\end{tabular}

\section{EXPERIMENTAL DESIGN AND ANALYSIS}

WEDM of eco-cut has been pre-owned to machine Austentic Stainless Steels AISI 316L and 317L with $\mathrm{P}_{\text {on }}$, $\mathrm{P}_{\text {off, }} \mathrm{V}$ and WT as input limitations. Varying the above limitations a cut of $10 \mathrm{~mm}$ length are fabricated on the work piece of two disparate materials. The aim of the present work is to optimize the WEDM procedure parameters for better performance [6],[7]. In this work the showing measures are material removal rate (MRR), surface roughness ( $\mathrm{Ra}$ ) and cutting speed (CS).

The level of input limitations is fixed based on the trial runs displayed in the work table, response surface methodologyBox Behnken Design method has been used for experimentation (Table III and Table IV). The measured and calculated responses are given in the work table after conducting the experiments. ANOVA [8],[9],[10] has been used to know the significant parameter and their contribution.

The trial run defines as in the following steps:

1. Selecting the machining limitations and levels of these limitations.

2. Conducting the trial runs at all feasible level of the combinations.

MRR was calculated using the following equation,

MRR calculation: $\mathrm{MRR}=[\mathrm{WLT}] / \mathrm{T}_{\mathrm{m}}$

$\mathrm{T}$ - THICKNESS OF WORK PIECE (mm),

L - LENGTH OF CUT (mm),

W - WIDTH OF CUT OR WIDTH (mm),

$\mathrm{T}_{\mathrm{m}}-$ MACHINING TIME (min)

$\operatorname{MRR}=(0.345)(10)(5) / 449=0.038 \mathrm{~mm}^{3} / \mathrm{min}$

MITUTOYO SURFTEST SJ 201P surface roughness tester is pre-owned to measure the surface roughness value $(\mathrm{Ra})$.

Table-III: Experimental Plan \& Responses of 316L

\begin{tabular}{|c|c|c|c|c|c|c|c|}
\hline & \multicolumn{4}{|c|}{ Inputs } & \multicolumn{3}{|c|}{ Outputs } \\
\hline $\begin{array}{l}\text { Run } \\
\text { order }\end{array}$ & $\begin{array}{l}P_{\text {on }} \\
(\mu s)\end{array}$ & $\begin{array}{l}P_{\text {off }} \\
(\mu \mathrm{s})\end{array}$ & $\begin{array}{l}V \\
\text { (V) }\end{array}$ & $\begin{array}{c}\text { WT } \\
(\mathrm{m} / \mathrm{min})\end{array}$ & $\begin{array}{l}\text { MRR } \\
(\mathbf{m m} / \\
\mathrm{min})\end{array}$ & $\begin{array}{c}\mathrm{CS} \\
(\mathrm{mm} / \\
\mathrm{min})\end{array}$ & $\begin{array}{c}\text { Ra } \\
(\mu \mathrm{m})\end{array}$ \\
\hline 1 & 112 & 44 & 22 & 10 & 0.025 & 3.07 & $\begin{array}{c}2.06 \\
69\end{array}$ \\
\hline 2 & 114 & 44 & 22 & 10 & 0.0384 & 3.01 & 2.12 \\
\hline 3 & 112 & 46 & 22 & 10 & 0.019 & 2.85 & $\begin{array}{c}1.96 \\
86\end{array}$ \\
\hline 4 & 114 & 46 & 22 & 10 & 0.0312 & 2.85 & 2.13 \\
\hline 5 & 113 & 45 & 20 & 9 & 0.035 & 3.12 & 1.95 \\
\hline 6 & 113 & 45 & 24 & 9 & 0.035 & 3.1 & 1.95 \\
\hline 7 & 113 & 45 & 20 & 11 & 0.0332 & 3 & 1.84 \\
\hline 8 & 113 & 45 & 24 & 11 & 0.038 & 3.15 & 2.27 \\
\hline 9 & 112 & 45 & 22 & 9 & 0.023 & 3.01 & 2.12 \\
\hline 10 & 114 & 45 & 22 & 9 & 0.0375 & 2.85 & 2.03 \\
\hline 11 & 112 & 45 & 22 & 11 & 0.025 & 3.1 & 2 \\
\hline 12 & 114 & 45 & 22 & 11 & 0.0365 & 2.62 & 2.19 \\
\hline 13 & 113 & 44 & 20 & 10 & 0.0365 & 3.17 & 2.06 \\
\hline 14 & 113 & 46 & 20 & 10 & 0.0259 & 3.12 & 1.85 \\
\hline 15 & 113 & 44 & 24 & 10 & 0.036 & 3.2 & 2.06 \\
\hline 16 & 113 & 46 & 24 & 10 & 0.0315 & 2.85 & 2.24 \\
\hline 17 & 112 & 45 & 20 & 10 & 0.022 & 2.99 & 1.95 \\
\hline 18 & 114 & 45 & 20 & 10 & 0.0410 & 2.49 & 1.99 \\
\hline 19 & 112 & 45 & 24 & 10 & 0.0301 & 2.85 & 2.24 \\
\hline 20 & 114 & 45 & 24 & 10 & 0.0375 & 2.44 & 2.24 \\
\hline 21 & 113 & 44 & 22 & 9 & 0.0324 & 2.18 & 2 \\
\hline 22 & 113 & 46 & 22 & 9 & 0.0296 & 2.88 & 1.96 \\
\hline 23 & 113 & 44 & 22 & 11 & 0.0635 & 3.18 & 2.1 \\
\hline 24 & 113 & 46 & 22 & 11 & 0.025 & 3.07 & 2 \\
\hline 25 & 113 & 45 & 22 & 10 & 0.037 & 2.7 & 2.07 \\
\hline 26 & 113 & 45 & 22 & 10 & 0.037 & 2.7 & 2.07 \\
\hline 27 & 113 & 45 & 22 & 10 & 0.037 & 2.7 & 2.07 \\
\hline
\end{tabular}

Table-IV: Experimental Plan and Responses of 317L

\begin{tabular}{|c|c|c|c|c|c|c|l|}
\hline & \multicolumn{4}{|c|}{ Inputs } & \multicolumn{3}{|c|}{ Outputs } \\
\hline $\begin{array}{c}\text { Run } \\
\text { order }\end{array}$ & $\begin{array}{c}\mathrm{P}_{\text {on }} \\
(\mu \mathrm{s})\end{array}$ & $\begin{array}{c}\mathrm{P}_{\text {off }} \\
(\mu \mathrm{s})\end{array}$ & $\begin{array}{c}\mathrm{V} \\
(\mathrm{V})\end{array}$ & $\begin{array}{c}\mathrm{WT} \\
(\mathrm{m} / \mathrm{min})\end{array}$ & $\begin{array}{c}\mathrm{MRR} \\
(\mathrm{mm} / \\
\mathrm{min})\end{array}$ & $\begin{array}{c}\mathrm{CS} \\
(\mathrm{mm} / \\
\mathrm{min})\end{array}$ & $\begin{array}{c}\mathrm{Ra} \\
(\mu \mathrm{m})\end{array}$ \\
\hline 1 & 112 & 44 & 22 & 10 & 0.0455 & 2.53 & 2.12 \\
\hline 2 & 114 & 44 & 22 & 10 & 0.0549 & 3.35 & 2.12 \\
\hline 3 & 112 & 46 & 22 & 10 & 0.0508 & 2.387 & 1.83 \\
\hline 4 & 114 & 46 & 22 & 10 & 0.0511 & 3.71 & 2.07 \\
\hline 5 & 113 & 45 & 20 & 9 & 0.062 & 3.15 & 2.25 \\
\hline 6 & 113 & 45 & 24 & 9 & 0.0573 & 3.21 & 2.10 \\
\hline 7 & 113 & 45 & 20 & 11 & 0.065 & 3.2 & 2.22 \\
\hline 8 & 113 & 45 & 24 & 11 & 0.055 & 3.10 & 2 \\
\hline 9 & 112 & 45 & 22 & 9 & 0.042 & 2.67 & $\begin{array}{l}1.19 \\
1\end{array}$ \\
\hline 10 & 114 & 45 & 22 & 9 & 0.0456 & 3.67 & 2.01 \\
\hline 11 & 112 & 45 & 22 & 11 & 0.041 & 2.58 & 1.78 \\
\hline 12 & 114 & 45 & 22 & 11 & 0.0477 & 3.71 & 2.06 \\
\hline 13 & 113 & 44 & 20 & 10 & 0.061 & 3.17 & $\begin{array}{l}2.31 \\
5\end{array}$ \\
\hline 14 & 113 & 46 & 20 & 10 & 0.068 & 3.21 & 2.29 \\
\hline
\end{tabular}




\begin{tabular}{|l|l|l|l|l|l|l|l|}
\hline 15 & 113 & 44 & 24 & 10 & 0.065 & 3.12 & 2.16 \\
\hline 16 & 113 & 46 & 24 & 10 & 0.068 & 3.31 & $\begin{array}{l}2.18 \\
1\end{array}$ \\
\hline 17 & 112 & 45 & 20 & 10 & 0.047 & 2.84 & 2.17 \\
\hline 18 & 114 & 45 & 20 & 10 & 0.04 & 3.63 & 2.06 \\
\hline 19 & 112 & 45 & 24 & 10 & 0.039 & 2.44 & 1.63 \\
\hline 20 & 114 & 45 & 24 & 10 & 0.0573 & 3.89 & 1.95 \\
\hline 21 & 113 & 44 & 22 & 9 & 0.062 & 3.15 & 2.19 \\
\hline 22 & 113 & 46 & 22 & 9 & 0.069 & 3.25 & 2.3 \\
\hline 23 & 113 & 44 & 22 & 11 & 0.059 & 3.04 & 2.18 \\
\hline 24 & 113 & 46 & 22 & 11 & 0.069 & 3.26 & 2.28 \\
\hline 25 & 113 & 45 & 22 & 10 & 0.0452 & 2.69 & 1.99 \\
\hline 26 & 113 & 45 & 22 & 10 & 0.0452 & 2.69 & 1.99 \\
\hline 27 & 113 & 45 & 22 & 10 & 0.0452 & 2.69 & 1.99 \\
\hline
\end{tabular}

\section{RESULTS AND DISCUSSION}

ANOVA has been applied on the exploratory out-comes for MRR, CS, Ra for both steels 316L and 317L are given Table V, Table VI, Table VII, Table VIII, Table IX and Table X respectively. By using surface optimizer, optimum level has been observed and measured as shown from Fig. 2 to Fig. 7.

Table-V: Outcomes of ANOVA for MRR 316L

\begin{tabular}{|c|c|c|c|c|c|c|}
\hline Source & DF & ss & MS & F-Value & $\begin{array}{c}\text { P- } \\
\text { Value }\end{array}$ & $\begin{array}{c}\% \\
\text { Contribut } \\
\text { ion }\end{array}$ \\
\hline Model & 14 & $\begin{array}{c}0.0009 \\
30\end{array}$ & $\begin{array}{c}0.00 \\
0066\end{array}$ & 443.53 & $\begin{array}{c}0.00 \\
0\end{array}$ & $\begin{array}{c}99.785 \\
40\end{array}$ \\
\hline A & 1 & $\begin{array}{c}0.0004 \\
99\end{array}$ & $\begin{array}{c}0.000 \\
499\end{array}$ & 3330.45 & $\begin{array}{c}0.00 \\
0\end{array}$ & $\begin{array}{c}53.655 \\
91\end{array}$ \\
\hline B & 1 & $\begin{array}{c}0.0001 \\
50 \\
\end{array}$ & $\begin{array}{l}0.000 \\
150 \\
\end{array}$ & 1004.32 & $\begin{array}{c}0.00 \\
0\end{array}$ & $\begin{array}{c}16.129 \\
03 \\
\end{array}$ \\
\hline C & 1 & $\begin{array}{c}0.0000 \\
19\end{array}$ & $\begin{array}{c}0.000 \\
019\end{array}$ & 127.72 & $\begin{array}{c}0.00 \\
0\end{array}$ & $\begin{array}{c}2.0430 \\
11\end{array}$ \\
\hline D & 1 & $\begin{array}{c}0.0000 \\
00\end{array}$ & $\begin{array}{c}0.000 \\
000\end{array}$ & 1.39 & $\begin{array}{c}0.26 \\
1 \\
\end{array}$ & 0 \\
\hline$A^{2}$ & 1 & $\begin{array}{c}0.0001 \\
04\end{array}$ & $\begin{array}{c}0.000 \\
104\end{array}$ & 694.20 & $\begin{array}{c}0.00 \\
0\end{array}$ & $\begin{array}{c}11.182 \\
8\end{array}$ \\
\hline $\mathbf{B}^{2}$ & 1 & $\begin{array}{c}0.0001 \\
03\end{array}$ & $\begin{array}{c}0.000 \\
103\end{array}$ & 685.15 & $\begin{array}{c}0.00 \\
0\end{array}$ & $\begin{array}{c}11.075 \\
27\end{array}$ \\
\hline $\mathrm{C}^{2}$ & 1 & $\begin{array}{c}0.0000 \\
00\end{array}$ & $\begin{array}{c}0.000 \\
000\end{array}$ & 0.02 & $\begin{array}{c}0.89 \\
9\end{array}$ & 0 \\
\hline $\mathbf{D}^{2}$ & 1 & $\begin{array}{c}0.0000 \\
20\end{array}$ & $\begin{array}{c}0.000 \\
020\end{array}$ & 132.94 & $\begin{array}{c}0.00 \\
0\end{array}$ & $\begin{array}{c}2.1505 \\
38\end{array}$ \\
\hline A*B & 1 & $\begin{array}{c}0.0000 \\
00\end{array}$ & $\begin{array}{c}0.000 \\
000\end{array}$ & 2.18 & $\begin{array}{c}0.16 \\
6\end{array}$ & 0 \\
\hline $\mathbf{A}^{*} \mathbf{C}$ & 1 & $\begin{array}{c}0.0000 \\
38\end{array}$ & $\begin{array}{c}0.000 \\
038\end{array}$ & 254.45 & $\begin{array}{c}0.00 \\
0\end{array}$ & $\begin{array}{c}4.0860 \\
22\end{array}$ \\
\hline$A^{*} \mathbf{D}$ & 1 & $\begin{array}{c}0.0000 \\
02\end{array}$ & $\begin{array}{c}0.000 \\
002\end{array}$ & 15.01 & $\begin{array}{c}0.00 \\
2\end{array}$ & $\begin{array}{c}0.2150 \\
54\end{array}$ \\
\hline $\mathbf{B}^{*} \mathbf{C}$ & 1 & $\begin{array}{c}0.0000 \\
19\end{array}$ & $\begin{array}{c}0.000 \\
019\end{array}$ & 62.38 & $\begin{array}{c}0.00 \\
0\end{array}$ & $\begin{array}{c}0.9677 \\
42\end{array}$ \\
\hline $\mathbf{D} * \mathbf{B}$ & 1 & $\begin{array}{c}0.0000 \\
19\end{array}$ & $\begin{array}{c}0.000 \\
019\end{array}$ & 126.62 & $\begin{array}{c}0.00 \\
0\end{array}$ & $\begin{array}{c}2.0430 \\
11\end{array}$ \\
\hline C*D & 1 & $\begin{array}{c}0.0000 \\
06\end{array}$ & $\begin{array}{c}0.000 \\
006\end{array}$ & 37.96 & $\begin{array}{c}0.00 \\
0\end{array}$ & $\begin{array}{c}0.6451 \\
61\end{array}$ \\
\hline Error & 12 & $\begin{array}{c}0.0000 \\
02\end{array}$ & $\begin{array}{r}0.000 \\
00015\end{array}$ & & & $\begin{array}{c}0.2150 \\
54\end{array}$ \\
\hline Total & 26 & & & & & 100 \\
\hline
\end{tabular}

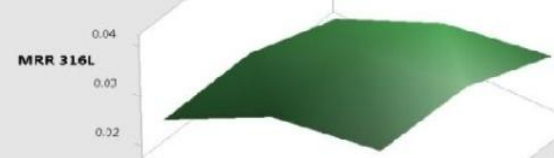

MRR for 316L $=-72.25+1.0589 \mathrm{~A}+0.4286 \mathrm{~B}+0.1345 \mathrm{C}$ $+0.2084 \mathrm{D}-0.004416 \mathrm{~A}^{2}-0.004388 \mathrm{~B}^{2}+0.000005 \mathrm{C}^{2}-$ $0.001933 \mathrm{D}^{2}-0.000286 \mathrm{~A} * \mathrm{~B}-0.001544 \mathrm{~A} * \mathrm{C}-0.000750$ $A * D+0.000764 B * C-0.002178 B * D+0.000596 C * D$

Table-VI: Outcomes of ANOVA for CS 316L

\begin{tabular}{|c|c|c|c|c|c|c|}
\hline Source & DF & SS & MS & F-Value & P-Value & \% Contribution \\
\hline Model & 14 & $\begin{array}{c}0.00 \\
093\end{array}$ & $\begin{array}{c}0.00 \\
0066\end{array}$ & 443.53 & 0.000 & 87.8251723 \\
\hline A & 1 & $\begin{array}{c}0.11 \\
288\end{array}$ & $\begin{array}{c}0.112 \\
889\end{array}$ & 11.53 & 0.005 & 11.6989481 \\
\hline B & 1 & $\begin{array}{c}0.11 \\
842\end{array}$ & $\begin{array}{c}0.118 \\
425\end{array}$ & 12.10 & 0.005 & 12.2726566 \\
\hline $\mathbf{C}$ & 1 & $\begin{array}{c}0.00 \\
163\end{array}$ & $\begin{array}{c}0.001 \\
633\end{array}$ & 0.17 & 0.690 & 0.16923157 \\
\hline $\mathbf{D}$ & 1 & $\begin{array}{c}0.00 \\
002\end{array}$ & $\begin{array}{c}0.000 \\
021\end{array}$ & 0.00 & 0.964 & 0.002176288 \\
\hline $\mathbf{A}^{2}$ & 1 & $\begin{array}{c}0.00 \\
066\end{array}$ & $\begin{array}{c}0.000 \\
664\end{array}$ & 0.07 & 0.799 & 0.06881186 \\
\hline $\mathbf{B}^{2}$ & 1 & $\begin{array}{c}0.26 \\
011\end{array}$ & $\begin{array}{c}0.260 \\
112\end{array}$ & 26.57 & 0.000 & 26.9560081 \\
\hline $\mathbf{C}^{2}$ & 1 & $\begin{array}{c}0.13 \\
117\end{array}$ & $\begin{array}{c}0.131 \\
175\end{array}$ & 13.40 & 0.003 & 13.5939686 \\
\hline $\mathbf{D}^{2}$ & 1 & $\begin{array}{c}0.21 \\
296\end{array}$ & $\begin{array}{c}0.212 \\
969\end{array}$ & 21.75 & 0.001 & 22.07047 \\
\hline $\mathbf{A}^{*} \mathbf{B}$ & 1 & $\begin{array}{c}0.00 \\
083\end{array}$ & $\begin{array}{c}0.000 \\
838\end{array}$ & 0.09 & 0.775 & 0.08684388 \\
\hline $\mathbf{A}^{*} \mathbf{C}$ & 1 & $\begin{array}{c}0.07 \\
022\end{array}$ & $\begin{array}{c}0.070 \\
225\end{array}$ & 7.17 & 0.020 & 7.27757915 \\
\hline $\mathbf{A}^{* \mathbf{D}}$ & 1 & $\begin{array}{c}0.02 \\
496\end{array}$ & $\begin{array}{c}0.024 \\
964\end{array}$ & 2.55 & 0.136 & 2.58707705 \\
\hline $\mathbf{B}^{*} \mathbf{C}$ & 1 & $\begin{array}{c}0.02 \\
250\end{array}$ & $\begin{array}{c}0.022 \\
500\end{array}$ & 2.30 & 0.155 & 2.33172703 \\
\hline $\mathbf{D}^{*} \mathbf{B}$ & 1 & $\begin{array}{c}0.00 \\
902\end{array}$ & $\begin{array}{c}0.009 \\
025\end{array}$ & 0.92 & 0.356 & 0.93528162 \\
\hline $\mathbf{C}^{*} \mathbf{D}$ & 1 & $\begin{array}{c}0.00 \\
722\end{array}$ & $\begin{array}{c}0.007 \\
225\end{array}$ & 0.74 & 0.407 & 0.74874346 \\
\hline Error & 12 & $\begin{array}{c}0.11 \\
748\end{array}$ & $\begin{array}{c}0.009 \\
790\end{array}$ & & & 12.1751386 \\
\hline Total & 26 & & & & & 100 \\
\hline
\end{tabular}

$\mathrm{CS}$ for $316 \mathrm{~L}=500+1.11 \mathrm{~A}-21.26 \mathrm{~B}-7.73 \mathrm{C}+2.32 \mathrm{D}-$ $0.0112 \mathrm{~A} * \mathrm{~A}+0.2208 \mathrm{~B} * \mathrm{~B}+0.0392 \mathrm{C} * \mathrm{C}+0.1998 \mathrm{D} * \mathrm{D}+$ $0.0145 \mathrm{~A} * \mathrm{~B}+0.0663 \mathrm{~A} * \mathrm{C}-0.0790 \mathrm{~A} * \mathrm{D}-0.0375 \mathrm{~B} * \mathrm{C}+$ $0.0475 \mathrm{~B} * \mathrm{D}+0.0213 \mathrm{C} * \mathrm{D}$

Table-VII: Outcomes of ANOVA for Ra 316L

\begin{tabular}{|c|c|c|c|c|c|c|}
\hline Source & DF & SS & MS & F-Value & P-Value & $\begin{array}{c}\% \\
\text { Contribution }\end{array}$ \\
\hline Model & 14 & $\begin{array}{c}0.3181 \\
35\end{array}$ & $\begin{array}{c}0.0227 \\
24\end{array}$ & 16.09 & 0.000 & 94.94017 \\
\hline
\end{tabular}


Optimization of Machining Parameters for AISI 316L And 317L Austenitic Stainless Steels using Eco-Cut Wire-EDM Technique

\begin{tabular}{|c|c|c|c|c|c|c|}
\hline A & 1 & $\begin{array}{c}0.0108 \\
78\end{array}$ & $\begin{array}{c}0.0108 \\
78\end{array}$ & 7.70 & 0.017 & 3.246292 \\
\hline B & 1 & $\begin{array}{c}0.0055 \\
26\end{array}$ & $\begin{array}{c}0.0055 \\
26\end{array}$ & 3.91 & 0.071 & 1.649109 \\
\hline C & 1 & $\begin{array}{c}0.1539 \\
52\end{array}$ & $\begin{array}{c}0.1539 \\
52\end{array}$ & $\begin{array}{c}108.9 \\
9\end{array}$ & 0.000 & 45.94348 \\
\hline D & 1 & $\begin{array}{c}0.0122 \\
37\end{array}$ & $\begin{array}{c}0.0122 \\
37\end{array}$ & 8.66 & 0.012 & 3.651855 \\
\hline$A^{2}$ & 1 & $\begin{array}{c}0.0089 \\
62\end{array}$ & $\begin{array}{c}0.0089 \\
62\end{array}$ & 6.34 & 0.027 & 2.674505 \\
\hline $\mathbf{B}^{2}$ & 1 & $\begin{array}{c}0.0023 \\
54\end{array}$ & $\begin{array}{c}0.0023 \\
54\end{array}$ & 1.67 & 0.221 & 0.702498 \\
\hline$C^{2}$ & 1 & $\begin{array}{c}0.0005 \\
82\end{array}$ & $\begin{array}{c}0.0005 \\
82\end{array}$ & 0.41 & 0.533 & 0.173685 \\
\hline $\mathbf{D}^{2}$ & 1 & $\begin{array}{c}0.0078 \\
83\end{array}$ & $\begin{array}{c}0.0078 \\
83\end{array}$ & 5.58 & 0.036 & 2.352502 \\
\hline$A * \mathbf{B}$ & 1 & $\begin{array}{c}0.0029 \\
32\end{array}$ & $\begin{array}{c}0.0029 \\
32\end{array}$ & 2.08 & 0.175 & 0.874989 \\
\hline$A^{*} \mathbf{C}$ & 1 & $\begin{array}{c}0.0004 \\
00\end{array}$ & $\begin{array}{c}0.0004 \\
00\end{array}$ & 0.28 & 0.604 & 0.119371 \\
\hline $\mathbf{A}^{*} \mathbf{D}$ & 1 & $\begin{array}{c}0.0186 \\
60\end{array}$ & $\begin{array}{c}0.0186 \\
60\end{array}$ & 13.21 & 0.003 & 5.568653 \\
\hline $\mathrm{B}^{*} \mathrm{C}$ & 1 & $\begin{array}{c}0.0378 \\
69\end{array}$ & $\begin{array}{c}0.0378 \\
69\end{array}$ & 26.81 & 0.000 & 11.30114 \\
\hline D*B & 1 & $\begin{array}{c}0.0009 \\
00\end{array}$ & $\begin{array}{c}0.0009 \\
00\end{array}$ & 0.64 & 0.440 & 0.268585 \\
\hline$C^{*} \mathbf{D}$ & 1 & $\begin{array}{c}0.0462 \\
25\end{array}$ & $\begin{array}{c}0.0462 \\
25\end{array}$ & 32.73 & 0.000 & 13.7948 \\
\hline Error & 12 & $\begin{array}{c}0.0169 \\
50\end{array}$ & $\begin{array}{c}0.0014 \\
13\end{array}$ & & & 5.0583438 \\
\hline Total & 26 & & & & & 100 \\
\hline
\end{tabular}

\begin{tabular}{|c|c|c|c|c|c|c|}
\hline $\mathbf{A} \mathbf{C}$ & 1 & $\begin{array}{c}0.000 \\
160\end{array}$ & $\begin{array}{c}0.00 \\
0160\end{array}$ & 16.92 & 0.001 & 6.324111 \\
\hline $\mathbf{A} \mathbf{D}$ & 1 & $\begin{array}{c}0.000 \\
002\end{array}$ & $\begin{array}{c}0.00 \\
0002\end{array}$ & 0.25 & 0.623 & 0.079051 \\
\hline $\mathbf{B} \mathbf{C}$ & 1 & $\begin{array}{c}0.000 \\
004\end{array}$ & $\begin{array}{c}0.00 \\
0004\end{array}$ & 0.42 & 0.528 & 0.158103 \\
\hline $\mathbf{D} \mathbf{B}$ & 1 & $\begin{array}{c}0.000 \\
002\end{array}$ & $\begin{array}{c}0.00 \\
0002\end{array}$ & 0.24 & 0.635 & 0.079051 \\
\hline $\mathbf{C} \mathbf{D}$ & 1 & $\begin{array}{c}0.000 \\
007\end{array}$ & $\begin{array}{c}0.00 \\
0007\end{array}$ & 0.74 & 0.406 & 0.27668 \\
\hline Error & 12 & $\begin{array}{c}0.000 \\
114\end{array}$ & $\begin{array}{c}0.00 \\
0009\end{array}$ & & & 4.505929 \\
\hline Total & 26 & & & & & 100 \\
\hline
\end{tabular}

\section{Surface Plot of Ra $316 \mathrm{~L}$ vs B, A}

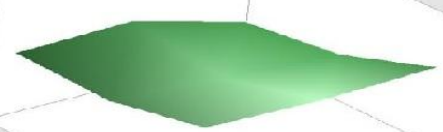

Fig. 4. Surface plot of Ra 316L vs. A and B

Ra for 316L $=730-11.03 \mathrm{~A}-2.11 \mathrm{~B}-1.99 \mathrm{C}-7.42 \mathrm{D}+$ $0.0410 \mathrm{~A} * \mathrm{~A}-0.0210 \mathrm{~B} * \mathrm{~B}-0.00261 \mathrm{C} * \mathrm{C}-0.0384 \mathrm{D} * \mathrm{D}+$ $0.0271 \mathrm{~A} * \mathrm{~B}-0.00500 \mathrm{~A} * \mathrm{C}+0.0683 \mathrm{~A} * \mathrm{D}+0.04865 \mathrm{~B} * \mathrm{C}-$ $0.0150 \mathrm{~B} * \mathrm{D}+0.05375 \mathrm{C} * \mathrm{D}$

Table-VIII: Outcomes of ANOVA for MRR 317L

\begin{tabular}{|c|c|c|c|c|c|c|}
\hline Source & DF & SS & MS & F-Value & $\begin{array}{c}\text { P- } \\
\text { Value }\end{array}$ & $\begin{array}{c}\% \\
\text { Contribution }\end{array}$ \\
\hline Model & 14 & $\begin{array}{c}0.002 \\
411\end{array}$ & $\begin{array}{c}0.00 \\
0172\end{array}$ & 18.21 & 0.000 & 95.29644 \\
\hline $\mathbf{A}$ & 1 & $\begin{array}{c}0.000 \\
081\end{array}$ & $\begin{array}{c}0.00 \\
0081\end{array}$ & 8.61 & 0.012 & 3.201581 \\
\hline $\mathbf{B}$ & 1 & $\begin{array}{c}0.000 \\
068\end{array}$ & $\begin{array}{c}0.00 \\
0068\end{array}$ & 7.18 & 0.020 & 2.68774 \\
\hline $\mathbf{C}$ & 1 & $\begin{array}{c}0.000 \\
000\end{array}$ & $\begin{array}{c}0.00 \\
0000\end{array}$ & 0.02 & 0.898 & 0 \\
\hline $\mathbf{D}$ & 1 & $\begin{array}{c}0.000 \\
000\end{array}$ & $\begin{array}{c}0.00 \\
0000\end{array}$ & 0.01 & 0.912 & 0 \\
\hline $\mathbf{A}^{2}$ & 1 & $\begin{array}{c}0.000 \\
295\end{array}$ & $\begin{array}{c}0.00 \\
0295\end{array}$ & 31.19 & 0.000 & 11.66008 \\
\hline $\mathbf{B}^{2}$ & 1 & $\begin{array}{c}0.000 \\
865\end{array}$ & $\begin{array}{c}0.00 \\
0865\end{array}$ & 91.48 & 0.000 & 34.18972 \\
\hline $\mathbf{C}^{2}$ & 1 & $\begin{array}{c}0.000 \\
331\end{array}$ & $\begin{array}{c}0.00 \\
0331\end{array}$ & 34.97 & 0.000 & 13.083 \\
\hline $\mathbf{D}^{2}$ & 1 & $\begin{array}{c}0.000 \\
234\end{array}$ & $\begin{array}{c}0.00 \\
0234\end{array}$ & 24.75 & 0.000 & 9.2490 \\
\hline $\mathbf{A}^{*} \mathbf{B}$ & 1 & $\begin{array}{c}0.000 \\
021\end{array}$ & $\begin{array}{c}0.00 \\
0021\end{array}$ & 2.19 & 0.165 & 0.83004 \\
\hline
\end{tabular}

Published By:

Surface Plot of mrr $317 \mathrm{~L}$ vs B, A

Fig. 5. Surface plot of MRR 317L vs. A and B MRR for 317L $=-71.1+1.709 \mathrm{~A}-0.883 \mathrm{~B}-0.4149 \mathrm{C}-$ $0.239 \mathrm{D}-0.00744 \mathrm{~A} * \mathrm{~A}+0.01274 \mathrm{~B} * \mathrm{~B}+0.001969 \mathrm{C} * \mathrm{C}+$ $0.00663 \mathrm{D} * \mathrm{D}-0.00228 \mathrm{~A} * \mathrm{~B}+0.003162 \mathrm{~A} * \mathrm{C}+0.00078$ $A * D-0.000500 B * C+0.00075 B * D-0.000662 C * D$

Table-IX: Outcomes of ANOVA for CS 317L

\begin{tabular}{|c|c|c|c|c|c|c|}
\hline Source & DF & SS & MS & F-Value & $\begin{array}{c}\text { P- } \\
\text { Value }\end{array}$ & $\begin{array}{c}\% \\
\text { Contribution }\end{array}$ \\
\hline Model & 14 & $\begin{array}{c}4.42 \\
615\end{array}$ & 0.31615 & 58.64 & 0.000 & 98.55929 \\
\hline $\mathbf{A}$ & 1 & $\begin{array}{c}3.55 \\
069\end{array}$ & 3.55069 & 658.57 & 0.000 & 79.06499 \\
\hline $\mathbf{B}$ & 1 & $\begin{array}{c}0.04 \\
843\end{array}$ & 0.04843 & 8.98 & 0.011 & 1.078415 \\
\hline $\mathbf{C}$ & 1 & $\begin{array}{c}0.00 \\
121\end{array}$ & 0.00121 & 0.22 & 0.644 & 0.026944 \\
\hline $\mathbf{D}$ & 1 & $\begin{array}{c}0.00 \\
350\end{array}$ & 0.00350 & 0.65 & 0.436 & 0.077936 \\
\hline $\mathbf{A}^{2}$ & 1 & $\begin{array}{c}0.17 \\
587\end{array}$ & 0.17587 & 32.62 & 0.000 & 3.916185 \\
\hline $\mathbf{B}^{2}$ & 1 & $\begin{array}{c}0.19 \\
577\end{array}$ & 0.19577 & 36.31 & 0.000 & 4.359308 \\
\hline $\mathbf{C}^{2}$ & 1 & $\begin{array}{c}0.44 \\
986\end{array}$ & 0.44986 & 83.44 & 0.000 & 10.01726 \\
\hline $\mathbf{D}^{2}$ & 1 & $\begin{array}{c}0.34 \\
797\end{array}$ & 0.34797 & 64.54 & 0.000 & 7.748422 \\
\hline $\mathbf{A}^{*} \mathbf{B}$ & 1 & $\begin{array}{c}0.06 \\
597\end{array}$ & 0.06597 & 12.24 & 0.004 & 1.468987 \\
\hline $\mathbf{A}^{*} \mathbf{C}$ & 1 & $\begin{array}{c}0.10 \\
890\end{array}$ & 0.10890 & 20.20 & 0.001 & 2.424931 \\
\hline $\mathbf{A}^{*} \mathbf{D}$ & 1 & $\begin{array}{c}0.00 \\
422\end{array}$ & 0.00422 & 0.78 & 0.393 & 0.093969 \\
\hline $\mathbf{C} \mathbf{*} \mathbf{D}$ & 1 & $\begin{array}{c}0.00 \\
563\end{array}$ & 0.00563 & 1.04 & 0.327 & 0.125366 \\
\hline $\begin{array}{c}0.00 \\
360\end{array}$ & 0.00360 & 0.67 & 0.430 & 0.080163 \\
\hline & 1 & 0.00599 & 1.11 & 0.313 & 0.133382 \\
\hline
\end{tabular}




\begin{tabular}{|c|c|c|c|c|c|c|}
\hline Error & 12 & $\begin{array}{c}0.06 \\
470\end{array}$ & 0.00539 & & & 1.440707 \\
\hline Total & 26 & & & & & 100 \\
\hline
\end{tabular}

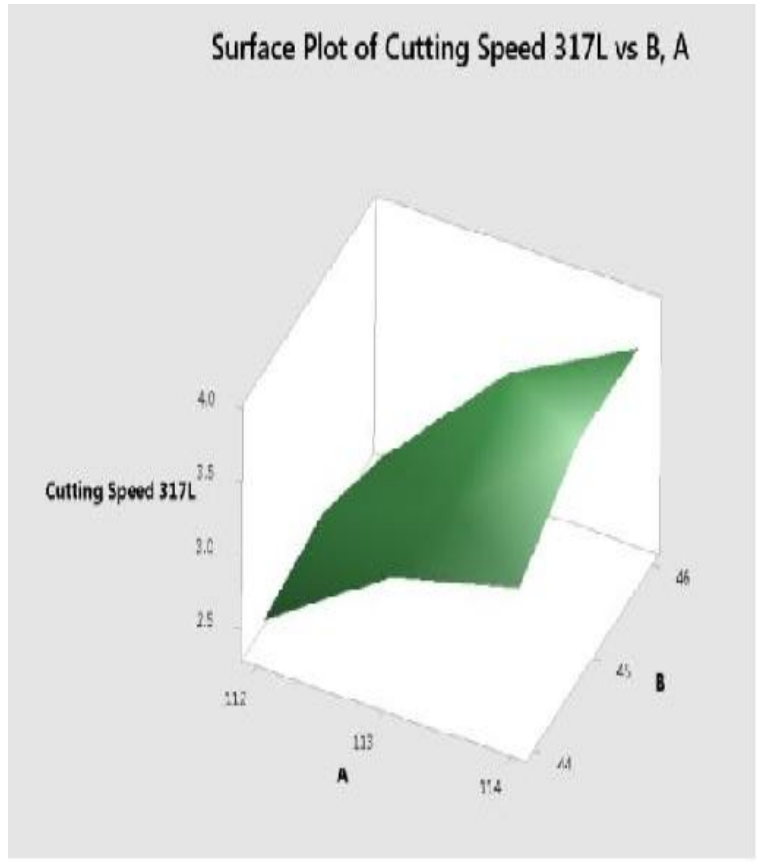

Fig. 6. Surface plot of CS 317L vs. A and $B$

CS for 317L $=3629-48.41 \mathrm{~A}-32.40 \mathrm{~B}-13.17 \mathrm{C}-9.72 \mathrm{D}$

$+0.1816 \mathrm{~A} * \mathrm{~A}+0.1916 \mathrm{~B} * \mathrm{~B}+0.07261 \mathrm{C} * \mathrm{C}+0.2554 \mathrm{D} * \mathrm{D}$

$+0.1284 \mathrm{~A} * \mathrm{~B}+0.0825 \mathrm{~A} * \mathrm{C}+0.0325 \mathrm{~A} * \mathrm{D}+0.0188 \mathrm{~B} * \mathrm{C}$

$+0.0300 \mathrm{~B} * \mathrm{D}-0.0193 \mathrm{C} * \mathrm{D}$

Table-X: Outcomes of ANOVA for Ra 317L

\begin{tabular}{|c|c|c|c|c|c|c|}
\hline Source & DF & ss & MS & $\begin{array}{c}\text { F- } \\
\text { Value }\end{array}$ & $\begin{array}{c}\text { P- } \\
\text { Value }\end{array}$ & $\begin{array}{c}\% \\
\text { Contribution }\end{array}$ \\
\hline Model & 14 & $\begin{array}{c}0.6602 \\
08\end{array}$ & $\begin{array}{c}0.04715 \\
8\end{array}$ & 8.98 & $\begin{array}{c}0.00 \\
0\end{array}$ & 91.2873 \\
\hline A & 1 & $\begin{array}{c}0.0587 \\
02\end{array}$ & $\begin{array}{c}0.05870 \\
2\end{array}$ & $\begin{array}{c}11.1 \\
8\end{array}$ & $\begin{array}{c}0.00 \\
6\end{array}$ & 8.116756 \\
\hline B & 1 & $\begin{array}{c}0.0013 \\
85\end{array}$ & $\begin{array}{c}0.00138 \\
5\end{array}$ & 0.26 & $\begin{array}{c}0.61 \\
7\end{array}$ & 0.191505 \\
\hline C & 1 & $\begin{array}{c}0.1371 \\
74\end{array}$ & $\begin{array}{c}0.13717 \\
4\end{array}$ & $\begin{array}{c}26.1 \\
2\end{array}$ & $\begin{array}{c}0.00 \\
0\end{array}$ & 18.96712 \\
\hline D & 1 & $\begin{array}{c}0.0048 \\
40\end{array}$ & $\begin{array}{c}0.00484 \\
0\end{array}$ & 0.92 & $\begin{array}{c}0.35 \\
6\end{array}$ & 0.669229 \\
\hline$A^{2}$ & 1 & $\begin{array}{c}0.0777 \\
42\end{array}$ & $\begin{array}{c}0.07774 \\
2\end{array}$ & $\begin{array}{c}14.8 \\
0\end{array}$ & $\begin{array}{c}0.00 \\
2\end{array}$ & 10.74943 \\
\hline $\mathbf{B}^{2}$ & 1 & $\begin{array}{c}0.1544 \\
36\end{array}$ & $\begin{array}{c}0.15443 \\
6\end{array}$ & $\begin{array}{c}29.4 \\
1\end{array}$ & $\begin{array}{c}0.00 \\
0\end{array}$ & 21.35394 \\
\hline$C^{2}$ & 1 & $\begin{array}{c}0.0338 \\
18\end{array}$ & $\begin{array}{c}0.03381 \\
8\end{array}$ & 6.44 & $\begin{array}{c}0.02 \\
6\end{array}$ & 4.676032 \\
\hline $\mathbf{D}^{2}$ & 1 & $\begin{array}{c}0.0289 \\
92\end{array}$ & $\begin{array}{c}0.02899 \\
2\end{array}$ & 5.52 & $\begin{array}{c}0.03 \\
7\end{array}$ & 4.008739 \\
\hline $\mathbf{A} * \mathbf{B}$ & 1 & $\begin{array}{c}0.0142 \\
92\end{array}$ & $\begin{array}{c}0.01429 \\
2\end{array}$ & 2.72 & $\begin{array}{c}0.12 \\
5\end{array}$ & 1.976162 \\
\hline $\mathbf{A}^{*} \mathbf{C}$ & 1 & $\begin{array}{c}0.0462 \\
25\end{array}$ & $\begin{array}{c}0.04622 \\
5\end{array}$ & 8.80 & $\begin{array}{c}0.01 \\
2\end{array}$ & 6.391554 \\
\hline$A^{*} \mathbf{D}$ & 1 & $\begin{array}{c}0.0081 \\
00\end{array}$ & $\begin{array}{c}0.00810 \\
0\end{array}$ & 1.54 & $\begin{array}{c}0.23 \\
8\end{array}$ & 1.119991 \\
\hline $\mathrm{B}^{*} \mathrm{C}$ & 1 & $\begin{array}{c}0.0005 \\
57\end{array}$ & $\begin{array}{c}0.00055 \\
7\end{array}$ & 0.11 & $\begin{array}{c}0.75 \\
0\end{array}$ & 0.077017 \\
\hline D*B & 1 & $\begin{array}{c}0.0000 \\
25\end{array}$ & $\begin{array}{c}0.00002 \\
5\end{array}$ & 0.00 & $\begin{array}{c}0.94 \\
6\end{array}$ & 0.003457 \\
\hline C*D & 1 & $\begin{array}{c}0.0012 \\
60\end{array}$ & $\begin{array}{c}0.00126 \\
0\end{array}$ & 0.24 & $\begin{array}{c}0.63 \\
3\end{array}$ & 0.174221 \\
\hline Error & 12 & $\begin{array}{c}0.0630 \\
13\end{array}$ & $\begin{array}{c}0.00525 \\
1\end{array}$ & & & 8.712840 \\
\hline Total & 26 & & & & & 100 \\
\hline
\end{tabular}

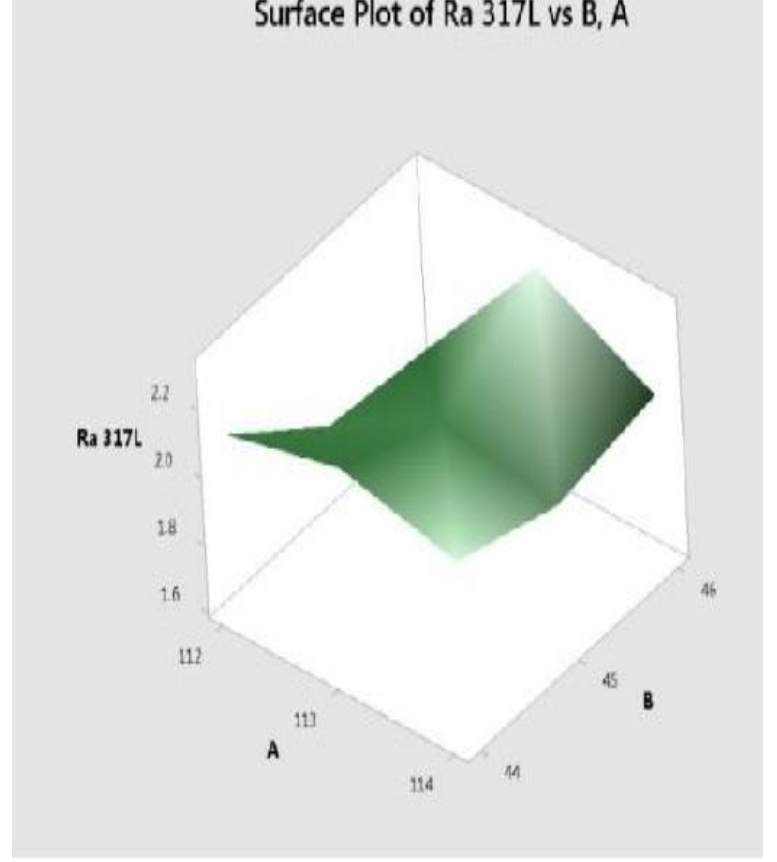

Fig. 7. Surface plot of Ra 317L vs. A and B

Ra for 317L $=-693+23.03 \mathrm{~A}-22.19 \mathrm{~B}-7.18 \mathrm{C}-6.27 \mathrm{D}-$ $0.1207 \mathrm{~A} * \mathrm{~A}+0.1702 \mathrm{~B} * \mathrm{~B}+0.01991 \mathrm{C} * \mathrm{C}+0.0737 \mathrm{D} * \mathrm{D}+$ $0.0598 \mathrm{~A} * \mathrm{~B}+0.0537 \mathrm{~A} * \mathrm{C}+0.0450 \mathrm{~A} * \mathrm{D}+0.0059 \mathrm{~B} * \mathrm{C}-$ $0.0025 \mathrm{~B} * \mathrm{D}-0.0089 \mathrm{C} * \mathrm{D}$

\section{CONCLUSIONS}

Response surface methodology (RSM) process has been used in the present work to optimize the WEDM performance measures [material removal rate-MRR cutting speed-CS and surface roughness-Ra]. Pulse on time $\left(\mathrm{P}_{\text {on }}\right)$, Pulse off time $\left(\mathrm{P}_{\text {off }}\right)$, Voltage $(\mathrm{V})$ and Wire tension(WT) have been considered as input limitations.The reaction of procedure limitations have been identified by registering ANOVA analysis for MRR, CS and Ra.

\section{For MRR, it is seen from ANOVA outcomes that,}

1. For 316L, $\mathrm{P}_{\text {on }}, \mathrm{P}_{\text {off }}, \mathrm{V}$, squares of $\mathrm{P}_{\text {on }}, \mathrm{P}_{\text {off }}, \mathrm{WT}$, interaction of $\mathrm{P}_{\text {on }} \& \mathrm{~V}, \mathrm{P}_{\text {off }} \& \mathrm{~V}, \mathrm{P}_{\text {off }} \& \mathrm{WT}, \mathrm{V} \& \mathrm{WT}$ are more influencing than other model terms.

2. For 317L, squares of $\mathrm{P}_{\text {on }}, \mathrm{P}_{\text {off }}$, interaction of $\mathrm{V} \&$ WT are more influencing than other model terms.

For CS it was develop from ANOVA outcomes that,

1. For 316L, squares of $\mathrm{P}_{\text {off }}$ are more influencing than other model terms.

2. For 317L, $\mathrm{P}_{\text {on }}$, squares of $\mathrm{P}_{\text {on }}, \mathrm{P}_{\text {off }}$, interaction of $\mathrm{V} \&$ WT are more influencing than other model terms.

For Ra it was develop from ANOVA outcomes that,

1. For 316L, V, interaction of $\mathrm{P}_{\text {off }} \& \mathrm{~V}, \mathrm{~V} \& \mathrm{WT}$ are more influencing than other model terms.

2. For 317L, $\mathrm{V} \&$ squares of $\mathrm{P}_{\text {off }}$ are more influencing than other model terms.

\section{REFERENCES}

1. F. Klocke, L. Hensgen, A. Klink, Ehle and Schwedt, "Structure and composition of the white layer In the Wire-EDM process," Procedia CIRP, vol. 42, 2016, pp. $673-678$.

2. S. Tilekar, S. S. Das and P. K. Patowari, "Process Parameter Optimization of Wire Edm On Aluminum And Mild Steel By Using Taguchi Method," Procedia Mater Sci, vol. 5, 2014, pp. 2577 -2584 .

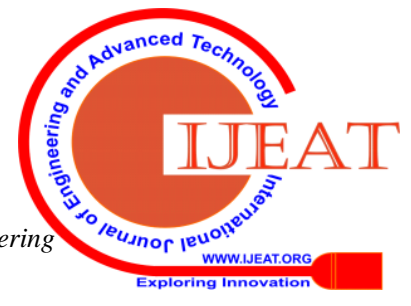


Optimization of Machining Parameters for AISI 316L And 317L Austenitic Stainless Steels using Eco-Cut Wire-EDM Technique

3. M. Durairaj, D. Sudharsun and N. Swamynathan, "Analysis of Process Limitations in Wire EDM with Stainless Steel using Single Objective Taguchi Method and Multi Objective Grey Relational Grade," Procedia Engg, vol. 64, 2013, pp. 868 - 877.

4. W. G. Bae, Kim, K. Y. Song, Jeong, Chong and Chu, "Engineering Stainless Steel Surface via Wire Electrical Discharge Machining for Controlling the Wet ability,"

Surface and Coatings Technol, vol. 275, 2015, pp. 316-323.

5. Y. Kaya and N. Kahraman, "An investigation into the explosive welding/cladding of Grade A ship steel/AISI 316L austenitic stainless steel," Mater and Des, vol. 52, 2013, pp. 367-372.

6. P. Raju, M. M. M. Sarcar and B. Satyanarayana, "Optimization of wire electric discharge machining limitations for surface roughness on $316 \mathrm{l}$ stainless steel using factorial experiment, " Procedia Mater Sci, vol. 5, 2014, pp. 1670-1676.

7. S. Sarkar, M. Sekh, S. Mitra , B. Bhattacharyya , "Modeling and optimization of wire electrical discharge machining of $\mathrm{TiAl}$ in trim cutting operation," J of Mater Process Technol, vol. 205, 2008, pp. 376-387.

8. C. Bhaskar Reddy, V. Diwakar Reddy and C. Eswara Reddy, "Experimental Investigations on Mrr And Surface Roughness of En 19 \& Ss 420 Steels In Wireedm Using Taguchi," Int J Engg Sci Technol, vol. 4, 2012, pp. 4603-4614.

9. Ching An Huang, Chwen Lin Shih, Kung Cheng Li and YauZen Chang., "The surface alloying behavior of martensitic stainless steel cut with wire electrical discharge machine," App Surface Sci, vol. 252, 2006, pp. 2915-2926.

10. C. A. Huang, F.Y. Hsu and S. J. Yao, "Microstructure analysis of the martensitic stainless steel surface fine-cut by the wire electrode discharge machining (WEDM)," Mater Sci Engg, vol. 371, 2004, pp. 119-126.

\section{AUTHORS PROFILE}

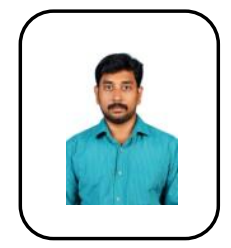

M.V.N. Srujan Manohar, is currently pursuing Ph.D. Full Time in the Department of Mechanical Engineering, Pondicherry Engineering College, Puducherry, India. He has five years of teaching experience.

Research Interests: Materials Technology

Email id: srujansujith8990@gmail.com

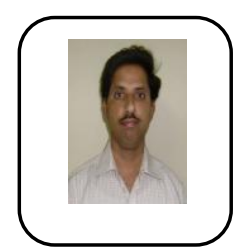

Dr. Y. Seetha Rama Rao, is currently working as a Associate Professor in the Department of Mechanical Engineering, GVPCOE(A), Visakhapatnam, India. He has completed his Ph.D. from JNTU University, Kakinada, Andhra Pradesh. He has more than 15 years of teaching experience. Research Interests: Machine Dynamics

Email id: yseetharamarao24@gvpce.ac.in

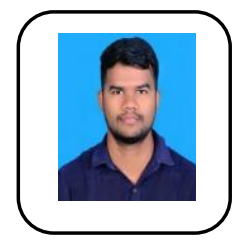

Ch. Sreeram, has completed his Post graduation degree in the field of CAD/CAM from the Department of Mechanical Engineering, GVPCOE(A), Visakhapatnam, India. Research Interests: Production Engineering

Email id: powermanu555@gmail.com 